\title{
Estudio numérico de la subsidencia causada por la excavación de túneles en un perfil de suelos blandos
}

CIVIL ENGINEERING

\section{Numerical study of settlements induced by tunneling in soft soil layers}

\author{
Adolfo C. Torres*, Gina C. Zambrano* $§$ \\ *Facultad de ingeniería. Programa de ingeniería civil. Universidad de La Salle. Bogotá, Colombia. \\ §atorresc@unisalle.edu.co,gina_5145@hotmail.com
}

(Recibido: Abril 15 de 2011- Aceptado: Noviembre 19 de 2012)

\begin{abstract}
Resumen
Este artículo presenta el desarrollo de una investigación numérica realizada con el fin de estudiar el comportamiento del terreno en superficie debido a la construcción de túneles en suelos blandos, específicamente para el caso de una estratigrafía típica de la zona aluvial de Bogotá. Para determinar la relación entre la subsidencia y la excavación de túneles construidos con máquina tuneladora (TBM - Tunneling Boring Machine) en éste tipo de suelo, se empleó la plataforma de elementos finitos PLAXIS 3D TUNNEL®.

Se estudiaron los niveles de incidencia en el fenómeno de subsidencia de cinco factores: el Modelo constitutivo de los materiales del suelo, la profundidad de excavación, el rendimiento de excavación, el diámetro y el espesor de la estructura del túnel.

Como resultado de la investigación se concluye que los factores con mayor influencia en la magnitud de la subsidencia son el diámetro del túnel y la profundidad de excavación.
\end{abstract}

Palabras clave: Método de elementos finitos, PLAXIS, Subsidencia, Túneles

\begin{abstract}
This paper presents the development of a numerical study performed to analyze the ground surface behavior due to the construction of tunnels in soft soils, specifically in the case of typical stratigraphy of the alluvial zone of Bogotá. A finite element platform PLAXIS 3D TUNNEL ${ }^{\circledR}$ was used to determine the relationship between the settlement and the tunneling excavation with TBM in this type of soil.

The levels of impact in the settlement phenomenon were studied by five factors: the constitutive model of soil materials, the depth of excavation, the performance of the TBM, the diameter and thickness of the tunnel lining. The research concluded the following result: the most influential factors in the magnitude of the settlement phenomenon are the tunnel diameter and the depth of excavation.
\end{abstract}

Key words: Finite element method, PLAXIS, Settlement, Tunnel 


\section{Introducción}

Se entiende el fenómeno de subsidencia, como el hundimiento del suelo debido a las excavaciones de galerías, túneles, extracciones mineras o a la extracción de agua subterránea, lo cual ocasiona una pérdida en el volumen del suelo. Uno de los referentes que expresa adecuadamente el estado de arte en el estudio del fenómeno se observa en el trabajo de Leca (2007), donde se resalta que los movimientos del suelo en superficie ocasionados por la construcción de un túnel, dependen de la profundidad de la clave del túnel, las condiciones geológicas, hidrogeológicas y geotécnicas del terreno, la geometría del túnel y el método de construcción empleado.

La primera contribución más difundida en la explicación del fenómeno de subsidencia es probablemente la descrita por Schmidt (1969) quien sugiere adoptar una campana de Gauss invertida como ley transversal de asentamientos en la superficie del terreno. Peck (1969) ratifica, basándose en medidas obtenidas en observaciones de campo la geometría propuesta por Schmidt para la distribución de los asentamientos en superficie.
En el estudio de éste fenómeno es importante analizar los tres factores que definen la forma de la depresión: el desplazamiento máximo vertical $\left(\delta_{\max }\right)$, la amplitud de la campana de Gauss $(B)$ y el volumen de pérdida de terreno en superficie $\left(V_{d}\right)$, siendo éste último igual al área bajo la curva, como se muestra en la figura 1, y que se calcula usando la expresión de la Ec. (1)

$$
V_{d}=2.5 \cdot i \cdot \delta_{\max }
$$

El valor de $i$ es el punto de inflexión de la curva y se puede calcular, a la luz de los planteamientos de diferentes autores como Peck (1969), Clough \& Schmidt (1981), O’Reilly (1982) y Rankin (1987). Sagazeta \& Oteo (1987) simplificaron el tema para la idealización del fenómeno, considerando un túnel de sección circular, sin revestimientos, excavado en un suelo homogéneo, elástico e isotrópico en deformación plana, bajo este supuesto se obtiene la siguiente expresión simplificada para el máximo desplazamiento vertical, Ec. (2):

$$
\delta_{\max }=\frac{\gamma \cdot D^{2}}{E} \cdot\left(0,85-v_{s}\right)
$$

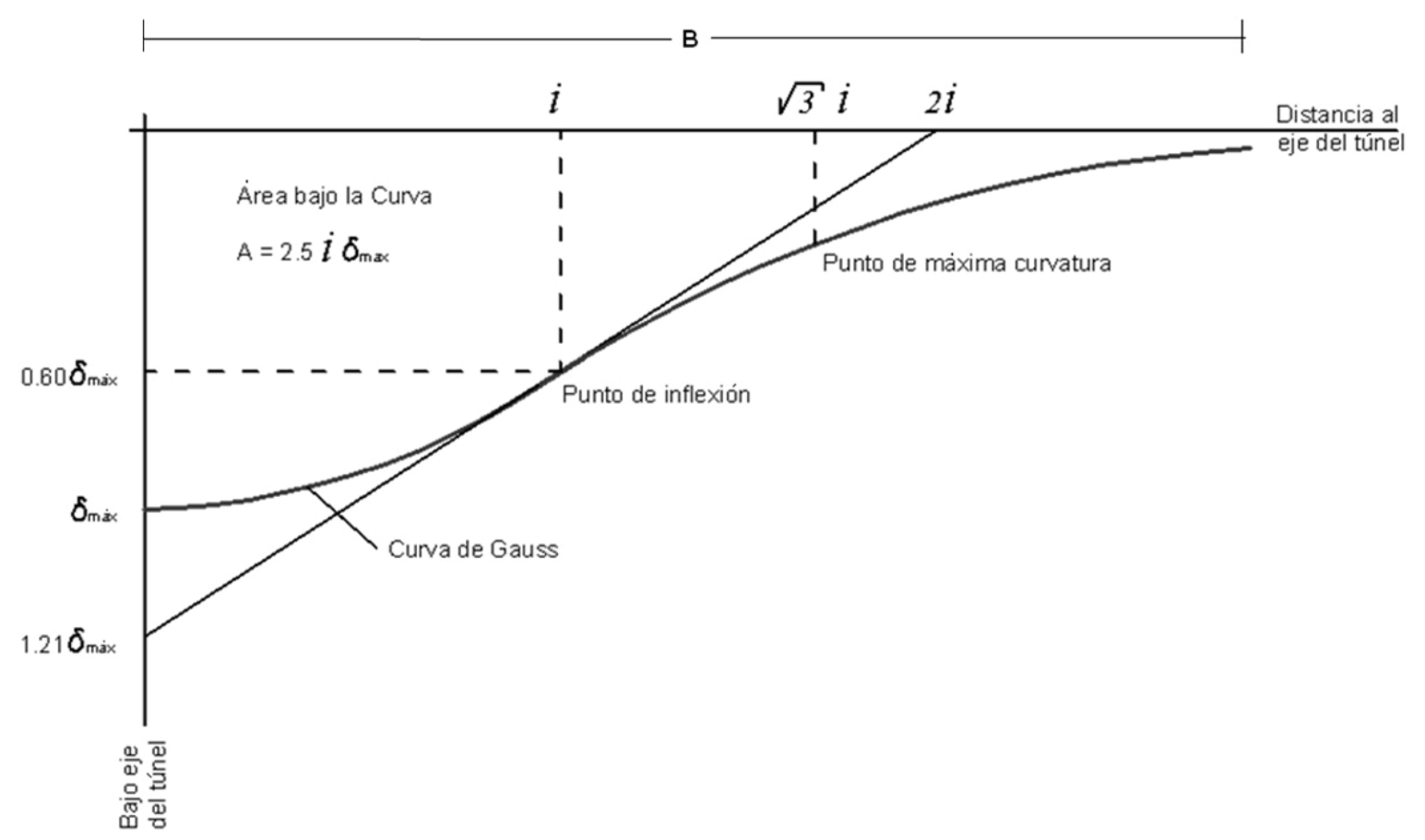

Figura 1. Relaciones geométricas en la curva de Gauss (idealización de la depresión en superficie producida por el fenómeno de subsidencia, Schmidt, 1969) 
Dónde: $\gamma$ es el peso unitario seco del terreno; $D$ el diámetro del túnel; $v_{s}$ el coeficiente de Poisson y $E$ es el módulo de elasticidad del terreno.

En los trabajos referidos anteriormente, se evidencia una importante influencia en el fenómeno de subsidencia por las variables de la profundidad a la clave del túnel y del diámetro del mismo. Es de anotar que todas las expresiones analíticas expresadas en esos trabajos son aproximaciones en condiciones geotécnicas muy particulares y se basan en observaciones de campo de múltiples proyectos, algunos trabajos más recientes como el de Yanga et al. (2004) presentan propuestas analíticas más elaboradas a partir de la teoría de medios estocásticos para la predicción de la subsidencia.

Estudios numéricos específicamente empleando el método de elementos finitos en el análisis de la subsidencia han sido presentados en diversos trabajos como el de Yong \& Chung (2007) y Hisatake (2011) donde se presentan modelos en 2D para el análisis del problema, y en trabajos como el de Ong et al. (2007) quienes desarrollan un modelo en 3D. A nivel colombiano en el trabajo de Torres \& Nieto (2009) se desarrollaron modelos en 3D, sin embargo es de destacar que todos los trabajos anteriores tienen como objeto determinar la geometría de la deformación de la superficie a partir de datos de entrada fijados por las condiciones específicas de los casos de estudio. Uno de los trabajos que aplicando métodos experimentales numéricos evalúan la incidencia de las variables que intervienen en el fenómeno de la subsidencia es el de Alean (2010) bajo la plataforma Phases2 v5.0, se realiza un análisis en $2 \mathrm{D}$ de la incidencia de la profundidad manteniendo constante el diámetro del túnel y los parámetros del suelo, como datos interesantes de los resultados del trabajo de Alean está el de corroborar que a mayor profundidad en el trazado de un túnel se van a presentar menores desplazamientos en la superficie del terreno y también pudo determinar que existe un valor de profundidad del túnel desde el cual la variación en el asentamiento máximo en superficie es muy bajo.

La presente investigación numérica se ha realizado con el fin de estudiar el comportamiento del terreno en superficie debido a la construcción de túneles en suelos blandos con el uso de TBM
(Tunnelling Boring Machine) y específicamente para un caso de tipología de suelos en Bogotá. Se estudiaron los niveles de incidencia en el fenómeno de subsidencia de cinco factores de interés: el Modelo constitutivo de los materiales del suelo, la profundidad de excavación, el rendimiento de la tuneladora, el diámetro del túnel y el espesor de dovelas del túnel. Dentro del contexto particular definido, también se busca dar un aporte inicial que contribuya a la predicción de los riesgos por la construcción de túneles en Bogotá, para poder planificar la construcción de estas importantes obras y así aportar en el desarrollo sustentable urbano aprovechando eficientemente el recurso del espacio subterráneo.

\section{Metodología}

A partir de los resultados de los trabajos dirigidos por Torres \& Nieto (2009) donde se desarrolla el estudio de subsidencia en un proyecto de túnel construido mediante TBM de presión balanceada de tierras EPB (Earth Presion Balance) que pasa por una estratigrafía típica de la zona aluvial de Bogotá; se construyó el esquema mostrado en la figura 2 que muestra la geometría básica del túnel, la posición del nivel freático y la estratigrafía del perfil del suelo que se incorporaron al modelo en elementos finitos. El trabajo de Torres \& Nieto (2009) permitió caracterizar los diferentes estratos de suelo y estableció por monitoreo en campo la medida promedio del asentamiento en superficie del terreno en el punto A que se indica en la figura 2 , siendo éste igual a $4,5 \mathrm{~cm}$.

El plan numérico experimental se plantea de la siguiente manera: se organizaron los factores de variación con un orden de importancia preliminar fijado por la revisión de los trabajos anteriormente referenciados y con una secuencia definida, las variaciones de cada uno de ellos se definen por un rango que en la presente investigación están dados por las dimensiones geométricas normales de túneles de transporte y por unos intervalos de variación dentro de los rangos que permitan una adecuado número de modelos, con los resultados finalmente de algunos parámetros de evaluación se realiza el análisis de incidencia de cada variable y una interpretación adecuada de los resultados. 


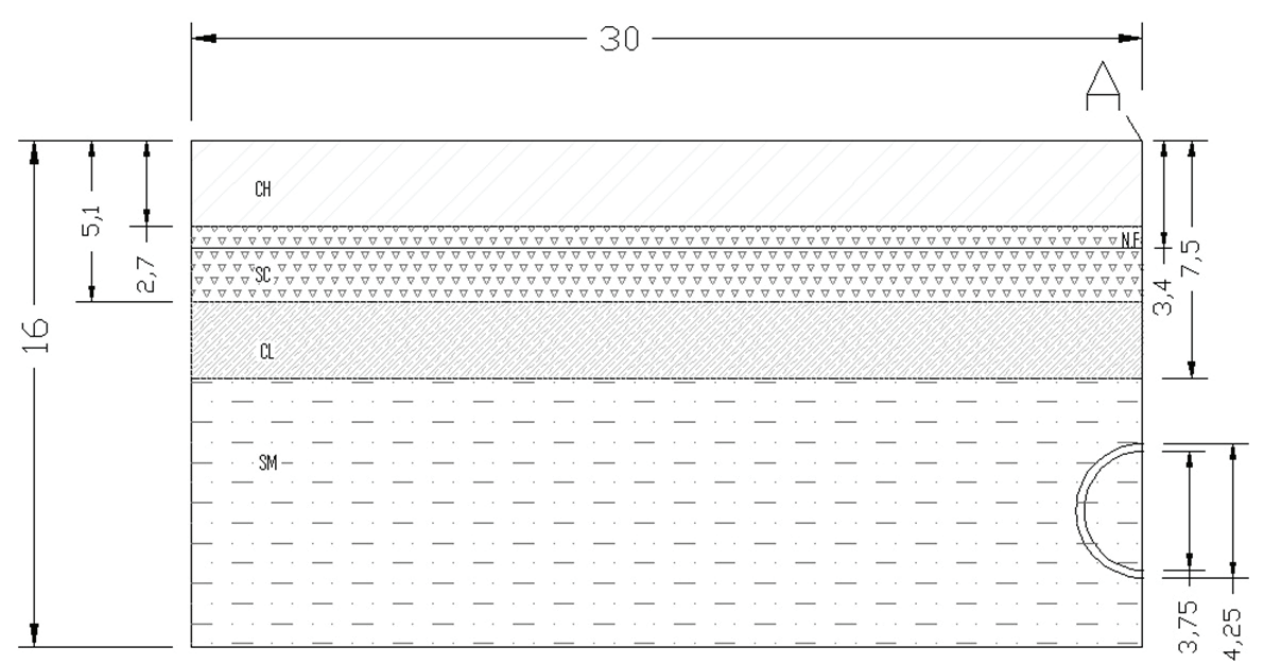

Figura 2. Esquema de cálculo

Así, los parámetros de evaluación de interés para ésta investigación y que permiten evaluar la incidencia de los factores de variación son los tres factores de forma de la curva de asentamientos: la deformación máxima vertical $\left(\delta_{\max }\right)$, la amplitud de la curva de subsidencia $(B)$ y el volumen de dicha depresión $\left(V_{d}\right)$. Se establecieron algunos factores de variación extraídos de las ecuaciones planteadas y trabajos de los autores nombrados anteriormente, como posibles factores de alta incidencia en las magnitudes de los parámetros de estudio, estos factores son cinco y en su orden son: el Modelo constitutivo de los materiales del suelo, la profundidad de excavación, el rendimiento de la tuneladora, el diámetro del túnel y el espesor de dovelas del túnel. Es de resaltar que tanto para el rendimiento de la tuneladora como para el espesor de dovelas no se encontró ninguna expresión o estudio previo de su incidencia en el fenómeno de subsidencia. Cada uno de los anteriores factores de variación tiene un código y un número de variantes definido por un intervalo de magnitudes, con lo anterior se definieron para la investigación numérica experimental un total de 27 modelos a calcular, en la tabla 1 se observa el plan numérico experimental.

Tabla 1. Plan numérico experimental

\begin{tabular}{|c|c|c|c|c|c|c|}
\hline FACTORES & $\begin{array}{l}\text { CÓDIGO } \\
\text { DE LA } \\
\text { VARIACIÓN }\end{array}$ & UNIDAD & $\begin{array}{l}\text { INTERVALO } \\
\text { DE LA } \\
\text { VARIACIÓN }\end{array}$ & $\begin{array}{l}\text { FACTOR DE } \\
\text { VARIACIÓN }\end{array}$ & $\begin{array}{l}\text { NÚMERO DE } \\
\text { VARIANTES } \\
(\mathrm{Kv})\end{array}$ & VALORES DE CÁLCULO \\
\hline $\begin{array}{c}\text { Modelos de } \\
\text { los materiales }\end{array}$ & $\mathrm{X} 1$ & ---- & $1-2$ & 1 & 2 & $\begin{array}{l}\text { Modelo de Mohr-Coulomb } \\
\text { Modelo de endurecimiento } \\
\text { del suelo }\end{array}$ \\
\hline Profundidad & $\mathrm{X} 2$ & $\mathrm{~m}$ & $15-60$ & 5 & 10 & $\begin{array}{c}15 ; 20 ; 25 ; 30 ; 35 ; 40 ; 45 \\
50 ; 55 ; 60\end{array}$ \\
\hline Rendimiento & $\mathrm{X} 3$ & $\mathrm{~m} /$ día & $25-45$ & 5 & 5 & $\begin{array}{l}\text { Para diámetros de } 3-6 \mathrm{~m} \\
\quad 25 ; 30 ; 35 ; 40 ; 45\end{array}$ \\
\hline Diámetro & $\mathrm{X} 4$ & $\mathrm{~m}$ & $3-6$ & 1 & 4 & $3 ; 4 ; 5 ; 6$ \\
\hline \multirow[t]{2}{*}{$\begin{array}{l}\text { Espesor de } \\
\text { la estructura } \\
\text { (pared) }\end{array}$} & $\mathrm{X} 5$ & $\mathrm{~m}$ & $0.25-0.50$ & 0.05 & 6 & $\begin{array}{c}0.25 ; 0.30 ; 0.35 ; 0.40 \\
0.45 ; 0.50\end{array}$ \\
\hline & & & \multicolumn{2}{|c|}{$\begin{array}{c}\text { TOTAL DE MODELOS A } \\
\text { REALIZAR }\end{array}$} & 27 & \\
\hline
\end{tabular}


En la fase preliminar de cálculos se creó un modelo basado en las condiciones geotécnicas observadas en terreno, y en la geometría del túnel en referencia de $3,75 \mathrm{~m}$ de diámetro interno, profundidad de $9,8 \mathrm{~m}$ y espesor de su estructura de $0,25 \mathrm{~m}$, inicialmente obteniendo un valor para $\delta_{\max }$ de $1,0 \mathrm{~cm}$. Mediante un ajuste menor de los parámetros medios del estrato del suelo $S M$ por donde pasa el túnel, pero obviamente manteniéndolos próximos a $39^{\circ}$ para el ángulo de fricción interna y $6 \mathrm{kPa}$ para la cohesión, valores medios reportados en los estudios de suelos (Torres \& Nieto, 2009) e incorporando un coeficientes de convergencia del escudo de la TBM que no se tenía registrado y que se fijo en un 5\% que es un valor normal para este tipo de equipos de excavación y de esta manera se logró en el cálculo del modelo base inicial obtener un valor de $\delta_{\max }$ igual a 4,4 $\mathrm{cm}$ de subsidencia en el punto A, obteniendo así un mínimo porcentaje de error con el desplazamiento vertical monitoreado en terreno que fue de $4,5 \mathrm{~cm}$. En la figura 3 se observa el modelo en 3D, las características geomecánicas de los suelos incorporados en el modelo se pueden ver en la tabla 2, y las propiedades de las estructuras tanto del escudo de perforación como del concreto del túnel en la tabla 3. Sobre éste modelo base se efectuaron las variaciones de los factores de posible incidencia, obteniendo de cada uno de los cálculos una curva para la subsidencia transversal sobre la cual se pueden analizar los tres factores de forma, de interés para ésta investigación.

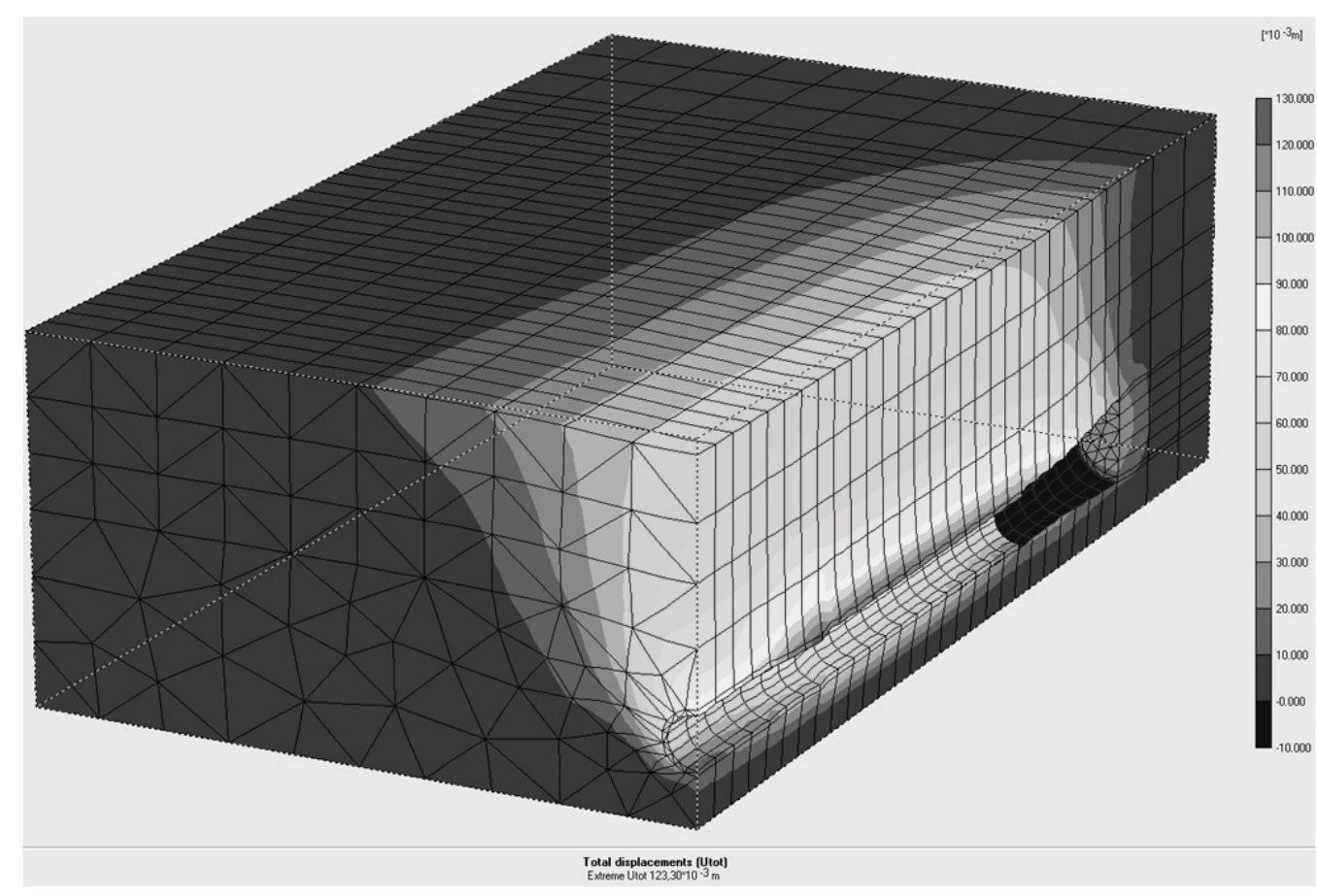

Figura 3. Vista del modelo ajustado en $3 D$

\section{Resultados y discusión}

\subsection{Incidencia del modelo constitutivo de los materiales en el fenómeno de subsidencia}

Cómo hipótesis inicial se creía que el modelo de los materiales no afectaría el resultado para el fenómeno de subsidencia, considerando que los diferentes modelos variarían el registro de la secuencia de la deformación durante la simulación del avance en la excavación, pero finalmente el desplazamiento máximo vertical final sería igual en cualquier caso.

Para esta serie de cálculos se conservaron las características geométricas del túnel y las condiciones hidrogeológicas y geotécnicas; sólo se modificó para cada cálculo el modelo constitutivo de los materiales del suelo por donde pasa el túnel es decir el estrato SM, en la tabla 4 se registran los parámetros de entrada para el modelo Hardening Soil. 
Como resultado de esta etapa de cálculos se registró una diferencia entre los modelos constitutivos del $10 \%$ en el desplazamiento máximo vertical y del $17 \%$ en el volumen de pérdida de terreno, en ambos casos, por exceso, en el modelo Hardening Soil. Ver figura 4. Sin embargo, teniendo en cuenta que los parámetros de entrada para el modelo constitutivo de endurecimiento del suelo se definieron mediante las correlaciones presentadas por Nieto et al. (2009), se estima que la diferencia de los resultados de cálculos para ésta serie no es significativa, por lo anterior los siguientes cálculos en la investigación experimental numérica se realizan bajo los lineamientos del modelo constitutivo de Mohr Coulomb.

Tabla 2. Parámetros de entrada de los suelos en el modelo Mohr Coulomb.

\begin{tabular}{ccccccc}
\hline PARÁMETRO & NOMBRE & CH & SC & CL & SM & UNIDAD \\
\hline $\begin{array}{c}\text { Modelo del material } \\
\text { Tipo de comportamiento } \\
\text { del material }\end{array}$ & Modelo & $\begin{array}{c}\text { Mohr }- \\
\text { Coulomb }\end{array}$ & $\begin{array}{c}\text { Mohr }- \\
\text { Coulomb }\end{array}$ & $\begin{array}{c}\text { Mohr }- \\
\text { Coulomb }\end{array}$ & $\begin{array}{c}\text { Mohr }- \\
\text { Coulomb }\end{array}$ & - \\
$\begin{array}{c}\text { Peso suelo sobre nivel } \\
\text { freático }\end{array}$ & $\gamma_{\text {unsat }}$ & 15,61 & 19,94 & 18,38 & 19,43 & $\mathrm{kN}^{2} \mathrm{~m}^{3}$ \\
$\begin{array}{c}\text { Peso suelo bajo nivel } \\
\text { freático }\end{array}$ & $\gamma_{\text {sat }}$ & 15,61 & 20,36 & 20,30 & 20,44 & $\mathrm{kN}^{2} \mathrm{~m}^{3}$ \\
$\begin{array}{c}\text { Módulo de Young } \\
\text { (Constante) }\end{array}$ & $E_{\text {ref }}$ & 3501 & 22089 & 6054 & 22089 & $\mathrm{kN} / \mathrm{m}^{2}$ \\
$\begin{array}{c}\text { Relación de Poisson } \\
\text { Cohesión (Constante) } \\
\text { Ángulo de fricción }\end{array}$ & $v$ & 0,35 & 0,3 & 0,35 & 0,3 & - \\
$\begin{array}{c}\text { Ángulo de dilatancia } \\
\text { Reducción de esfuerzo de } \\
\text { interface }\end{array}$ & $C_{\text {ref }}$ & 43,72 & 33,22 & 132,35 & 5 & $\mathrm{kN} / \mathrm{m}^{2}$ \\
$\begin{array}{c}\text { Coeficiente de presión } \\
\text { lateral de tierras }\end{array}$ & $R_{\text {inter }}$ & 13 & 22,88 & 19,64 & 35 & $\circ$ \\
\hline
\end{tabular}

Tabla 3. Propiedades de las estructuras en el modelo.

\begin{tabular}{cccc}
\hline \multicolumn{3}{c}{ Parámetros del concreto para el revestimiento del túnel } & \\
\hline PARÁMETRO & NOMBRE & VALOR & UNIDAD \\
Tipo de comportamiento & Tipo de material & Linear - elástico & - \\
Peso específico no saturado & $\gamma_{\text {unsat }}$ & 24,00 & $\mathrm{kN} / \mathrm{m}^{3}$ \\
Modulo elástico & $E_{\text {ref }}$ & $3100 \mathrm{E}+05$ & $\mathrm{kN} / \mathrm{m}^{2}$ \\
Relación de Poisson & $v$ & 0,100 & - \\
\hline & Parámetros del material del escudo de la TBM & $\mathrm{UNIDAD}$ \\
PARÁMETRO & NOMBRE & VALOR & \\
Tipo de comportamiento & Tipo de material & Elástico & $\mathrm{kN} / \mathrm{m}$ \\
Rigidez normal & $E A$ & $8,40 \mathrm{E}+08$ & $\mathrm{kNm} / \mathrm{m}$ \\
Espesor equivalente & $E I$ & 1.900 .000 & $\mathrm{~m}$ \\
Peso & $d$ & $5,21 \mathrm{E}-03$ & $\mathrm{kN} / \mathrm{m} / \mathrm{m}$ \\
Relación de Poisson & $w$ & 0,436 & - \\
\hline
\end{tabular}




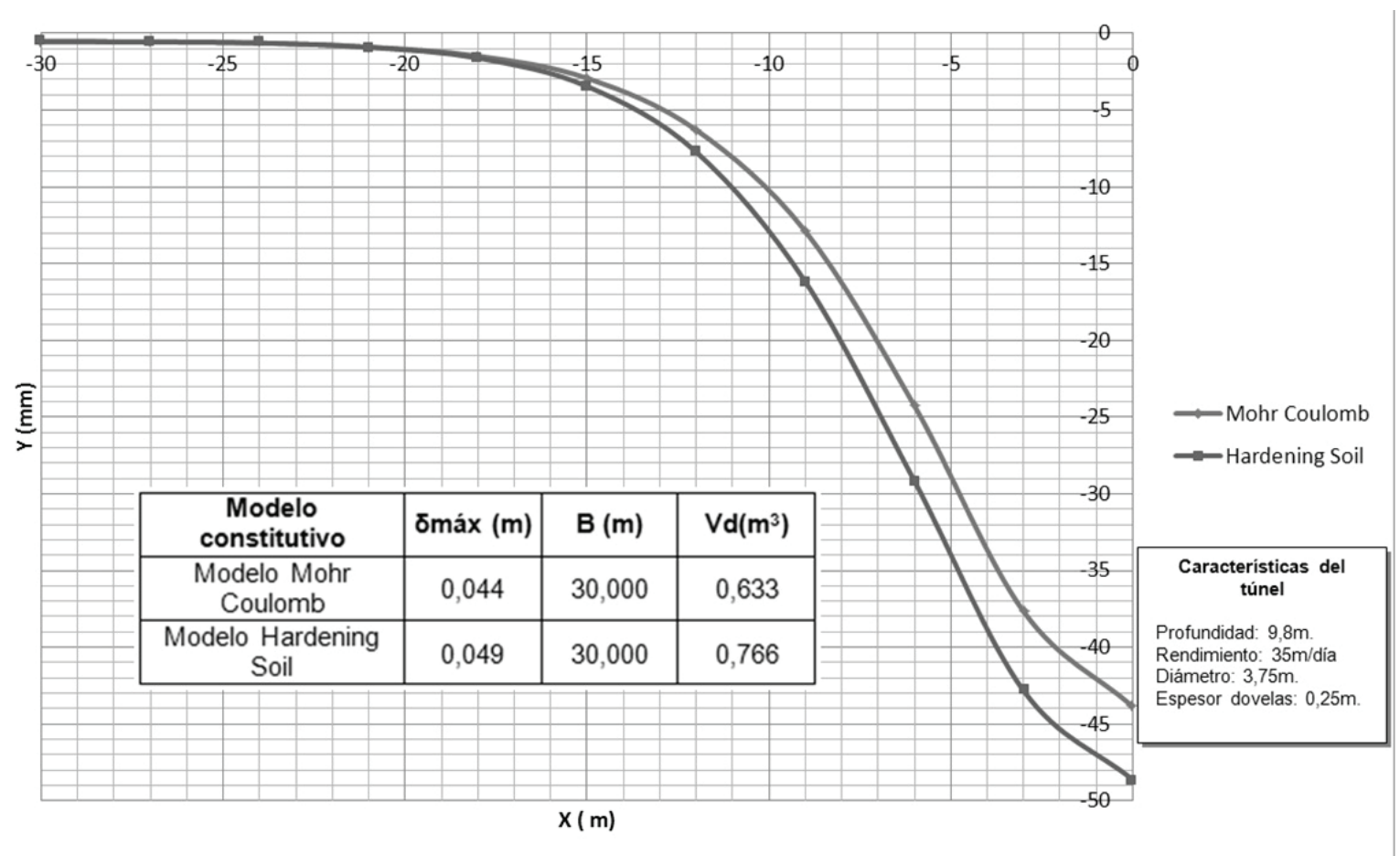

Figura 4. Incidencia del modelo constitutivo de los materiales en el fenómeno de subsidencia

Tabla 4. Parámetros de entrada modelo Hardening Soil.

\begin{tabular}{cc}
\hline Parámetros según la teoría de falla Mohr-Coulomb \\
\hline$c$ & $5 \mathrm{kN} / \mathrm{m}^{2}$ \\
$\varphi$ & $35^{\circ}$ \\
$\Psi$ & $5^{\circ}$ \\
\hline Parámetros básicos para la rigidez del suelo \\
\hline$E_{50}$ & $22089 \mathrm{kN} / \mathrm{m}^{2}$ \\
Eoed & $22089 \mathrm{kN} / \mathrm{m}^{2}$ \\
$M$ & 1 \\
\hline
\end{tabular}

3.2 Incidencia de la profundidad de excavación para túneles en el fenómeno de subsidencia

La profundidad es un factor de gran importancia ya que es un parámetro con alto grado de variación en los proyectos de túneles, de acuerdo al plan experimental establecido se realizaron 10 cálculos en esta serie. Para esta serie de cálculo fue preciso establecer la presión en el frente de excavación que aporta la TBM y que garantiza la mínima alteración en la condición de esfuerzos iniciales de la masa de suelo del frente, ésta presión es un parámetro dependiente de la profundidad de excavación, se utilizaron las cargas calculadas por Zambrano (2010).
En la figura 5, se puede ver que hay una profundidad desde la cual los asentamientos empiezan a tener tasas de variación muy bajas, en este caso en particular a una profundidad de 20 metros, (punto de máxima concavidad), este punto en la curva se relaciona con una profundidad óptima para reducir los asentamientos en superficie, con esta profundidad se continuo con las series de cálculos de los restantes parámetros en estudio, lo anterior concuerda con los resultados de Alean (2010). La profundidad tiene entonces, una relación inversamente proporcional con la subsidencia, teniendo un comportamiento asintótico a mayores profundidades.

Para el modelo base de ésta investigación, es decir el de menor profundidad, se registró el mayor desplazamiento vertical, siendo éste de 4,4 centímetros; mientras que para el modelo de mayor profundidad a 60 metros bajo la superficie, la subsidencia tuvo una magnitud de 1,6 centímetros, con base en lo anterior se estableció que el desplazamiento máximo vertical y la amplitud de la depresión registrados en superficie entre el mínimo y el máximo valor del rango de variación, tiene una diferencia del orden del $63 \%$, mientras que el 
volumen muestra una diferencia del orden del $38 \%$. En la figura 6 . Se observa que aunque los desplazamientos máximos verticales se reducen a una mayor profundidad, el área de impacto de la construcción de un túnel relacionada con el valor de $B$ es más amplia; por lo tanto, es necesario determinar una profundidad óptima a la cual si bien los asentamientos sean mínimos, también el área de impacto en la superficie sea la menor posible.

\section{PROFUNDIDAD (m) VS. $\delta(\mathrm{cm})$}

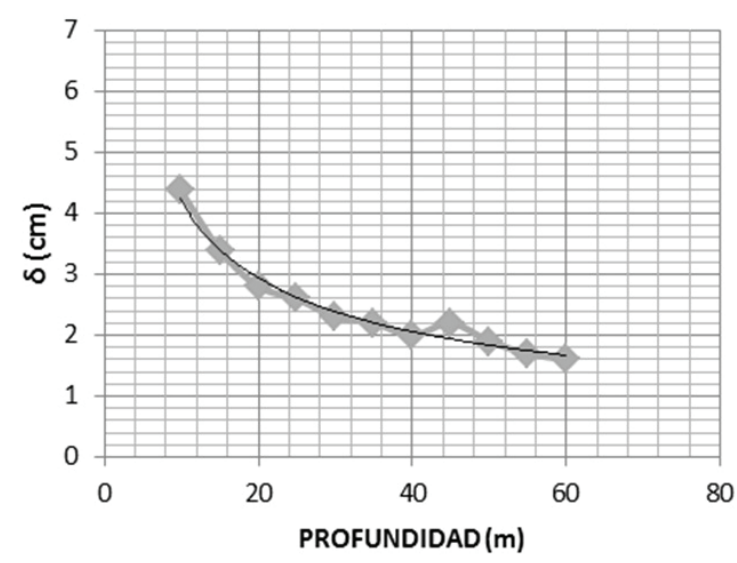

Figura 5. Incidencia del modelo constitutivo de los materiales en el fenómeno de subsidencia

\subsection{Incidencia del rendimiento de la tuneladora en el fenómeno de subsidencia}

El proyecto del túnel de referencia para el presente estudio se construyó empleando una máquina tuneladora de presión de tierras TBM - EPB. La hipótesis inicial que se tenía para considerar el rendimiento de la tuneladora como un posible factor de incidencia en el fenómeno de subsidencia, se basaba en que la variación del rendimiento afecta directamente la velocidad de aplicación de cargas en el frente de excavación, lo que podría afectar el volumen de pérdida de terreno en superficie o la condición de estados de esfuerzos iniciales, además la práctica mundial en la construcción de túneles reconoce que un avance rápido y constante en la construcción de túneles garantiza condiciones favorables de estabilidad en la obra.

La serie de cálculos para el rendimiento arrojo resultados exactamente iguales para todos los modelos en cuanto la forma de la campana de subsidencia. Lo que implica, que el rendimiento de la tuneladora tiene muy poca incidencia en el fenómeno de subsidencia; por lo tanto, se mantuvo contante con un valor de $35 \mathrm{~m} /$ día para todos los modelos siguientes en la investigación.
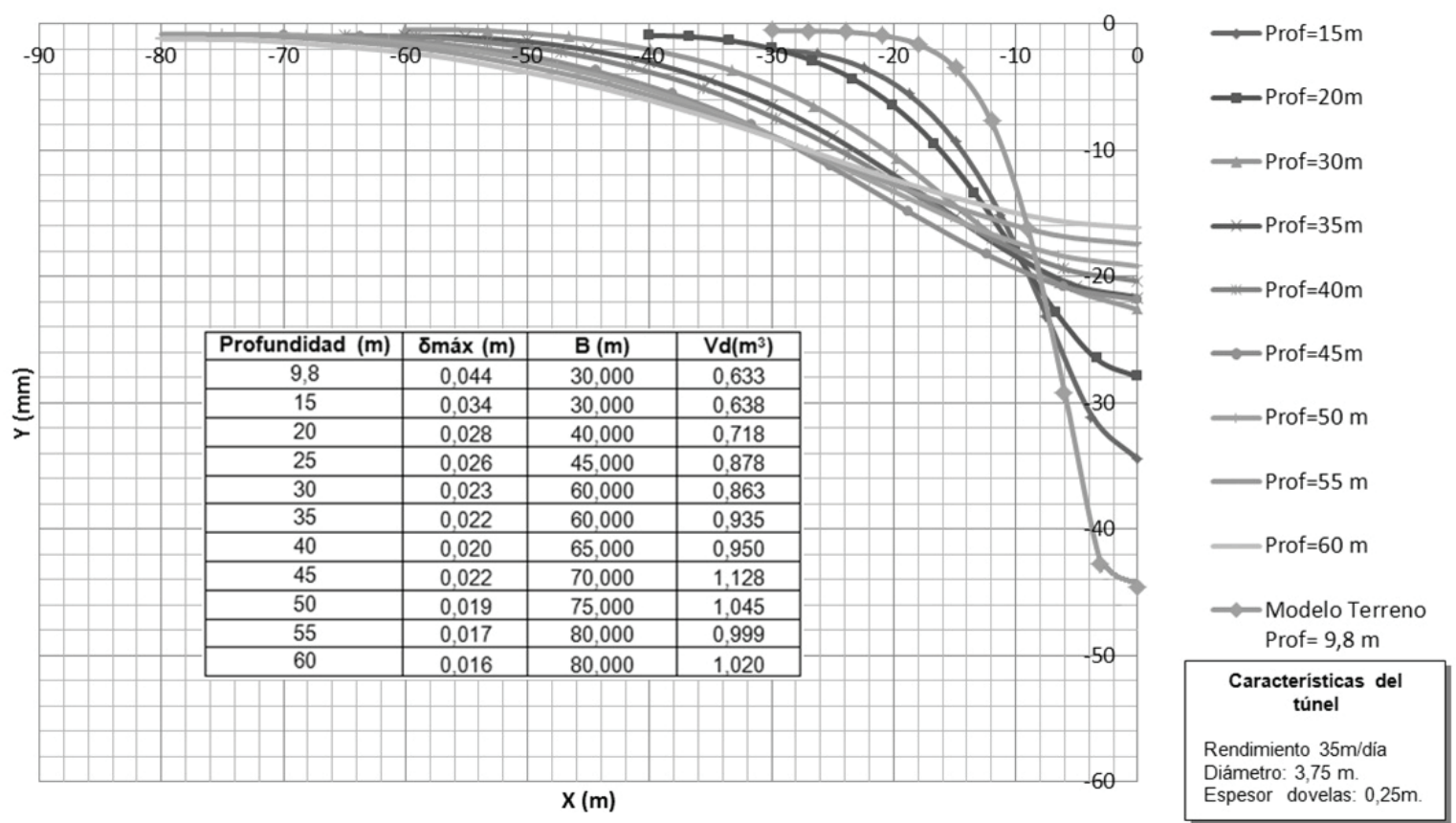

Figura 6. Efecto de la variación de la profundidad de excavación de túneles en el fenómeno de subsidencia. 


\subsection{Incidencia del diámetro del túnel en el fenómeno de subsidencia}

Los resultados de esta serie de cálculos se registran en la figura 7, donde se puede observar que el desplazamiento máximo para el punto de control crece linealmente, al incrementar el diámetro de excavación. Teniendo en cuenta que el siguiente factor de variación que se va a modelar es el espesor de la estructura, se decide dejar como diámetro base para los siguientes modelos el mayor diámetro es decir de $6 \mathrm{~m}$, dimensión que se relaciona con un valor medio para túneles de transporte y metros.

Lavariación del diámetro ocasiona una diferencia del $70 \%$ en el desplazamiento máximo vertical para el rango de variación, además la amplitud de la curva de subsidencia es mayor en un $62 \%$ para el diámetro de 6 metros en comparación con el mínimo valor de variación. Por otra parte si se analiza el volumen de pérdida de terreno en superficie, está en una relación muy cercana al $80 \%$ más de pérdida para el valor de diámetro de 6 metros.

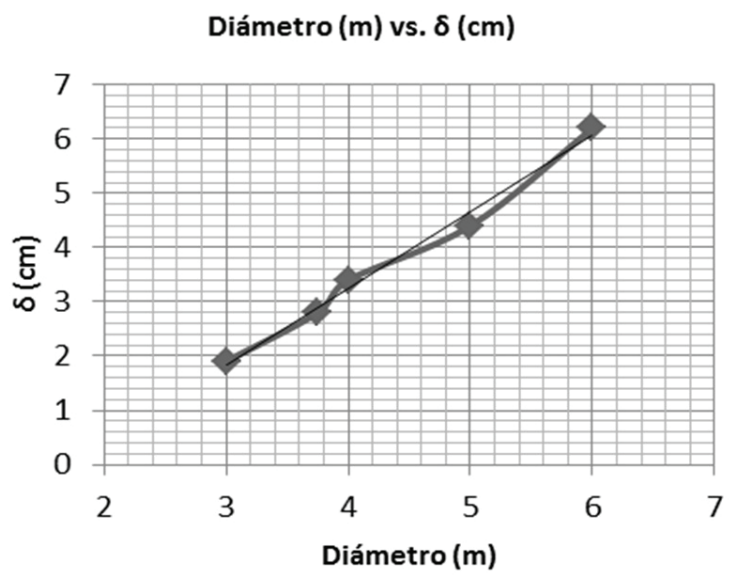

Figura 7. Incidencia del diámetro en el fenómeno de subsidencia

Con los estudios realizados en esta investigación se puede decir que el diámetro interno es un factor de importancia primaria para estudiar el fenómeno de subsidencia ya que registra curvas más amplias, ver figura 8 , generando asentamientos más altos, grandes áreas de influencia en superficie y los mayores volúmenes de pérdida de terreno.

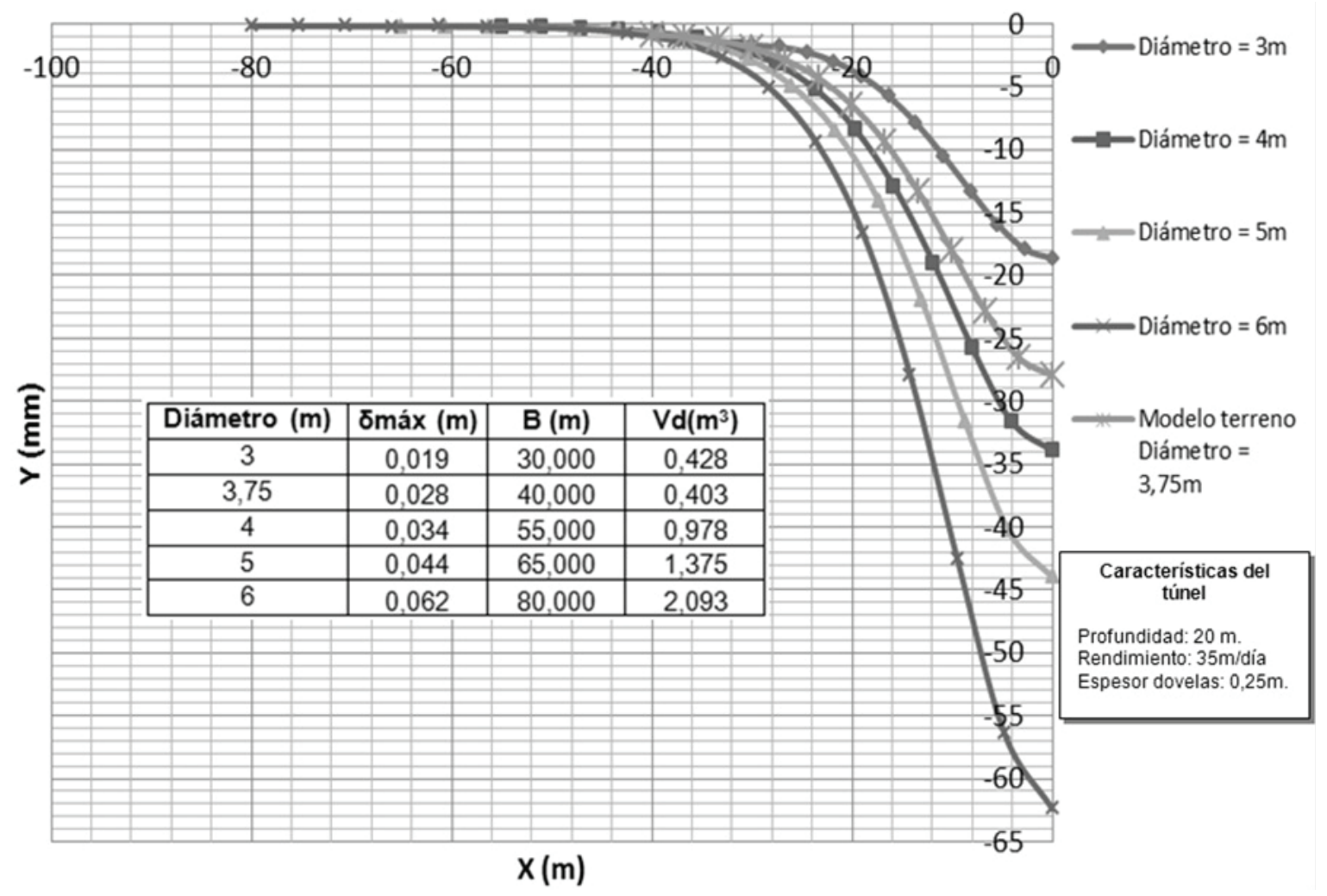

Figura 8. Efecto de la variación del diámetro interno de túneles en el fenómeno de subsidencia. 


\subsection{Incidencia del espesor de la estructura del túnel en el fenómeno de subsidencia}

En la figura 9, se puede analizar que debido a que el espesor de la estructura genera un incremento directo en el diámetro de excavación, este factor causa un efecto en la magnitud de los hundimientos en superficie.

Para los valores máximo y mínimo del rango de variación, el desplazamiento máximo vertical tiene una relación del $13 \%$, la amplitud de la campana de Gauss varía en un $14 \%$, mientras que el volumen de pérdida en el terreno mantiene una diferencia del $11 \%$ para los valores registrados. Ver figura 10.

Si bien es cierto que al aumentar el espesor de la estructura se afecta el fenómeno de subsidencia, debido a que el diámetro de excavación se aumenta también y como se señaló en el numeral anterior, el diámetro tiene una relación directamente proporcional con los desplazamientos en superficie, se puede establecer que el espesor de la estructura es la variable que registró menor incidencia en el fenómeno estudiado. Además es importante resaltar, que a diferencia del diámetro interno del túnel, el espesor de la estructura es un factor que se puede controlar, según las necesidades, con un valor adecuado de la resistencia del concreto.

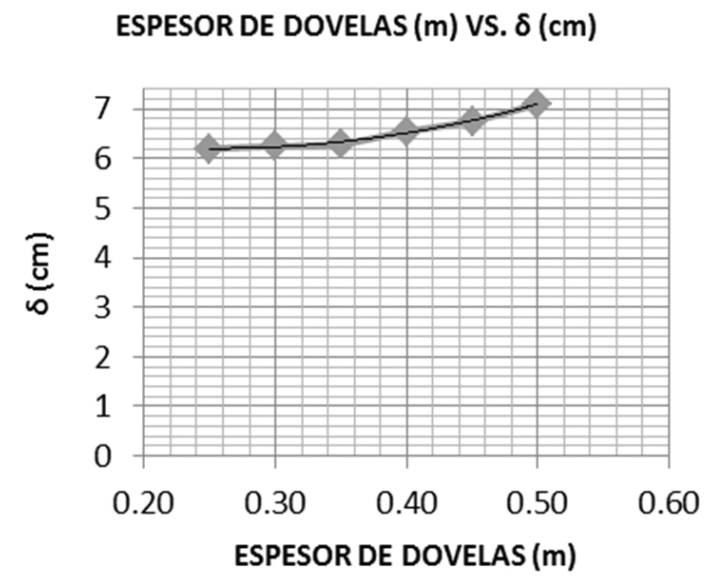

Figura 9. Incidencia del diámetro en el fenómeno de subsidencia

\section{Conclusiones}

Para el desarrollo de ésta investigación, se realizó un modelo base en $3 \mathrm{D}$ según las características del proyecto estudiado y monitoreado en el proyecto de investigación de Torres \& Nieto (2009), el modelo base se

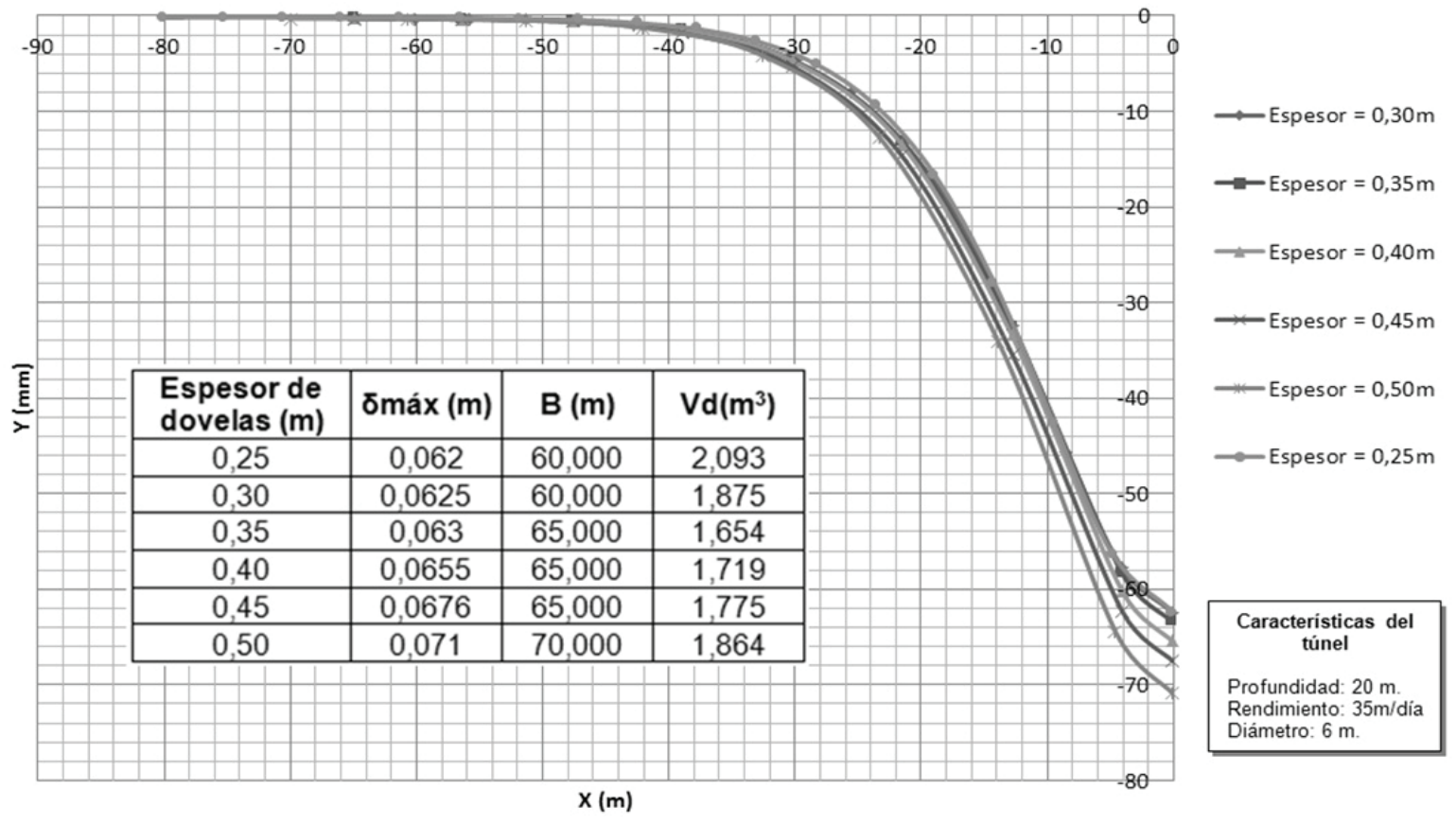

Figura 10. Efecto de la variación del espesor de la estructura en dovelas para túneles en el fenómeno de subsidencia. 
ajustó con un porcentaje de error del $2.5 \%$ para el valor de la subsidencia que se registró en el previo trabajo de campo, el cual constituye una base sólida para esta investigación. Teniendo en cuenta que el modelo inicial, sin ajustes mostraba una diferencia cercana al $400 \%$, se puede concluir, que los modelos numéricos por sí solos mantienen una elevada incertidumbre en sus resultados, por lo tanto para lograr una adecuada aproximación a la realidad, es importante apoyarse en trabajos de campo o modelaciones físicas para sus ajustes.

La profundidad de trazado de los túneles registró una incidencia importante en la subsidencia ocasionada. La relación entre la caída de terreno en superficie producida por la construcción de túneles en suelos blandos, y la profundidad de trazado de los mismos, es inversamente proporcional. Sin embargo, se debe encontrar la profundidad óptima para la cual, los asentamientos sean mínimos y que a su vez el área de influencia de la obra en superficie se reduzca.

Se pudo establecer que el diámetro interno del túnel es la variable con mayor incidencia en el fenómeno de estudio. La pérdida de volumen causada por el factor de variación del diámetro excede en un $50 \%$ a los registro por variación de la profundidad; sin embargo, es claro que la posible escala de variación del valor del diámetro del túnel es mucho menor que la de su profundidad.

Por otra parte el espesor de la estructura de revestimiento y el modelo constitutivo de los materiales para realizar la modelación en FEM, tienen una variación menor en la forma de la depresión en superficie, mientras que el rendimiento de la tuneladora no afecta los resultados de los modelos.

Durante la ejecución de ésta investigación, se constató la incidencia que tienen las condiciones de frontera en los resultados arrojados por los modelos, por lo tanto es necesario, en la creación de los modelos establecer una amplitud tal que no altere los resultados. Se recomienda realizar un cálculo inicial mediante las formulaciones analíticas del valor de $B$ y establecer la frontera de los modelos a dos veces el valor calculado.

El resultado de esta investigación se convierte en una base importante para avanzar en el desarrollo de planteamientos matemáticos con el fin de proponer una ecuación que contribuya a la evaluación rápida del fenómeno de subsidencia.

Esta investigación aporta la metodología para que en otros estudios de este estilo puedan estudiar el comportamiento de dicho fenómeno en otros tipos de suelo y se recomienda también como complemento a esta investigación, que en el futuro se efectué un análisis de la subsidencia en sentido longitudinal al eje del túnel.

\section{Referencias Bbibliográficas}

Alean, M. (2010). Deformaciones Inducidas por la construcción de túneles. Tesis Ingeniería Civil, Departamento de Ingeniería Civil y Agrícola, Universidad Nacional de Colombia, Bogotá, Colombia

Clough G., \& Schmidt B. (1981). Design and performance of excavation and tunnels in soft clay, Soft Clay Eng., Amsterdam: Elsevier, p. 569-634.

Hisatake M., (2011). A proposed methodology for analysis of ground settlements caused by tunneling, with particular reference to the "buoyancy" effect. Tunnelling and Underground Space Technology 26, 130-138.

Leca, E. (2007). Settlements induced by tunneling in Soft Ground. Tunnelling and Underground Space Technology 22, 119-149.

Nieto, A., Camacho, J., \& Ruiz, E. (2009). Determinación de parámetros para los modelos elastoplásticos Mohr-Coulomb y Hardening Soil en suelos arcillosos. Revista Ingenierías Universidad de Medellín 8 (15), 75-91.

Ong C.W., Leung C.F., Yong K.Y., \& Chow Y.K. (2007). Performance of pile due to tunnelinginduced soil movements. En: Underground Space - the 4th Dimension of Metropolises, Londres: Elsevier, p.619-624. 
O'Reilly, N. (1982). Settlements above tunnels in the U.K. In Proceedings Tunnelling 82. I.M.M. Londres, U.K.

Peck R. (1969). Deep Excavations and tunneling in soft ground, In Proceedings $7^{\circ}$ ICOSOMEF - ETAT Actuel des conaissances. México, p 225-259.

Rankin W., (1987). Ground movements resulting from urban tunneling: predictions and effects, In Proceedings 23rd Annual Conference of Eng. Geology of Underground Mov., Nottingham. U.K. Publication of: Geological Society, England p. 79-92

Sagazeta C., \& Oteo C., (1987). Análisis de subsidencia original por la excavación de túneles. En memorias del $1^{\mathrm{o}}$ Simposio Nacional sobre Túneles 1., Madrid, España. Vol. I, Comunicación II, 10

Schmidt B., (1969). Settlements and ground movements associated with tunneling in soil. Doctoral Thesis. University of Illinois. U.S.A. Torres, A., \& Nieto, F. (2009). Study of settlements induced by TBM in soft grounds in Bogotá-Colombia. In Safe Tunnelling for the city and for the environment - Proceedings of ITA-AITES World Tunnel Congress, Budapest, Hungría, p. 52-53.

Yanga J.S., Liua B.C., \& Wangb M.C., (2004). Modeling oftunneling-induced ground surface movements using stochastic medium theory. Tunnelling and Underground Space Technology 19, 113-123.

Yong-Joo Lee, Chung-SikYoo, (2007). Subsurface settlement profiles above shallow and deep model tunnels at large ground loss, Underground Space - the 4th Dimension of Metropolises, Londres: Elsevier, p.545-551.

Zambrano, G., (2010). Estudio numérico por medio de FEM de la relación: subsidencia vs. profundidad de excavación para túneles en un perfil de suelos blandos. Optando al título de Ingeniero Civil, Programa de Ingeniería Civil, Universidad de la Salle, Bogotá, Colombia. 